\title{
Design of Automatic Water Supply Upper Computer System
}

\author{
Peng Lu' ${ }^{1}$, Fengzhi Dai ${ }^{1,2}$ *, Tianyi Zhang ${ }^{1}$ \\ ${ }^{1}$ Tianjin University of Science and Technology, China; \\ ${ }^{2}$ Tianjin Tianke Intelligent and Manufacture Technology CO., LTD, China \\ E-mail: *daifz@tust.edu.cn \\ www.tust.edu.cn
}

\begin{abstract}
Aiming at the unstable water supply and fluctuating water pressure in the water supply system, an upper computer system of automatic water supply system based on Siemens PLC and KingView software was proposed. The pressure sensor in the water supply pipeline is used to detect the pressure in the pipeline, and the liquid level sensor monitors the liquid level in the tank. The sensor transmits the data to the PLC, and the PLC issues the control instruction after the computation processing. The KingView software can realize real-time monitoring and fault alarm of the system. The system can effectively improve the stability of water supply, and can avoid human entering the dangerous environment to search for unknown faults.
\end{abstract}

Keywords: KingView, constant pressure water supply, PLC, upper computer system, remote control

\section{Introduction}

With the continuous development of China's urbanization process, the pressure on the urban water supply system is increasing. In old urban communities, regional water supply is basically achieved by the municipal pipe network through secondary artificial pressurization, water towers and high-level pools.

However, these water supply methods have serious imbalances between water pressure and water consumption. In addition, the monitoring of parameters such as the pressure of the pipe network and the running state of the motor in the old-fashioned water supply system is through manual inspection of measuring instruments. This greatly reduces the operating safety of the operator. And when the water supply pressure changes greatly at different times, it can only be adjusted manually. This adjustment method is difficult to meet the system adjustment requirements in time ${ }^{1}$.

Based on the above discussion, this design focuses on the upper computer system for constant pressure water supply. The KingView software and Siemens PLC are used to design the upper computer of the water supply system and connect it with the lower computer of the water supply system. Finally, realize the constant pressure water supply, remote monitoring and remote operation of the water supply system.

\section{The Hardware Structure}

The core task of this system design is to remotely monitor and operate the water supply system through the host computer. Use PLC to make the frequency converter implement cyclic control of multiple pumps ${ }^{2}$. So the whole hardware system is composed of a liquid level sensor, three water pumps, a pressure sensor, a frequency converter and PLC.

\subsection{SIMATIC S7-300 PLC}

This design uses SIMATIC S7-300 PLC. SIMATIC S7-300 PLC has good versatility. It has the advantages of 
easy realization of distributed configuration, modularization, low user difficulty in mastering, and no exhaust fan structure. It is very suitable for the small-scale control ${ }^{3}$.

This design controls the frequency converter through PLC, and then controls the start and stop of the water pump through the frequency converter. SIMATIC S7-300 PLC is shown in Fig.1.

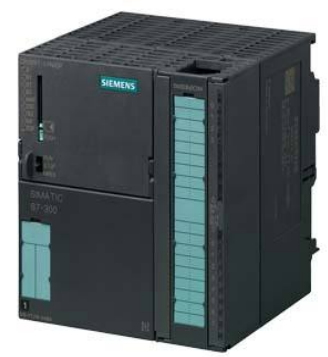

Fig.1. SIMATIC S7-300 PLC

The automatic water supply system has a medium amount of program and does not occupy much storage space. And the computing speed, communication resources and programming resources required by the system are not much. So this design chooses the CPU model of 315-2DP in SIMATIC S7-300 PLC. The 315-2DP used in this design is shown in Fig.2.This CPU has the following characteristics:

- $48 \mathrm{~KB}$ working memory.

- $0.3 \mathrm{~ms} / 1000$ instructions

- $\mathrm{MPI}+\mathrm{DP}$ connection.

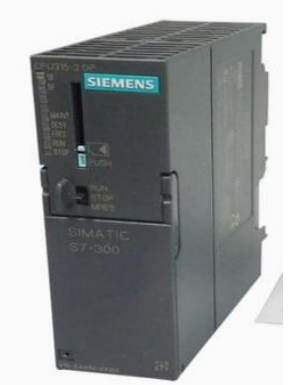

Fig.2. The 315-2DP CPU

\subsection{Frequency converter}

This design uses the MicroMaster430 series inverters, which are particularly suitable for driving water pumps and fans. The model used is 6SE6430-2UD27-5CA0. The power of this type of inverter is $7.5 \sim 250 \mathrm{KW}$. It is controlled by a microprocessor and has high functional diversity. MicroMaster430 series inverters are particularly suitable for driving water pumps and fans. Has high flexibility and reliability. The following functions can be realized: bypass function, multi-pump switching, energy-saving operation, manual/automatic switching, broken belt and water shortage detection, etc ${ }^{4}$. The inverter terminal interface is shown in Fig.3.

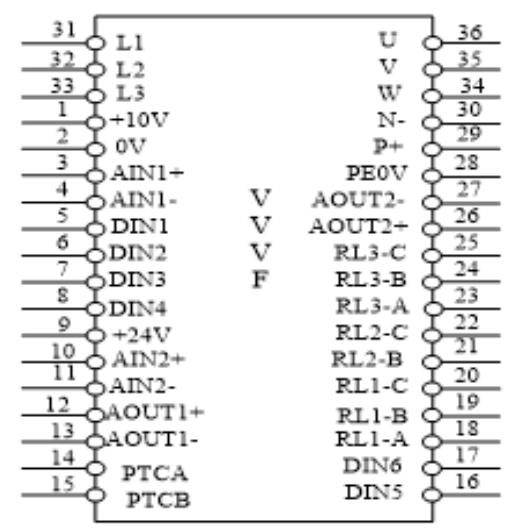

Fig.3. Inverter terminal interface

\subsection{Sensor module}

In this system design, $\mathrm{MC} 20 \mathrm{~B}$ economical pressure sensor and MC20C drop-in liquid level sensor are used.

The MC20B economical pressure sensor selected in this design can modify the range and zero point according to the needs on site. And it has high anti-interference and overload performance, and it is not prone to failure.

The MC20C drop-in liquid level sensor uses an isolated diffused silicon sensor to convert the hydrostatic pressure into an electrical signal. Then after temperature compensation and linear correction, it is converted into standard electrical signal output. The liquid level sensor has the following characteristics:

- Measuring range: $0.3 \mathrm{M} \sim 200 \mathrm{M}$.

- Working temperature: $-20{ }^{\circ} \mathrm{C} \sim 80{ }^{\circ} \mathrm{C}$.

- Output signal: $4 \mathrm{~mA} \sim 20 \mathrm{~mA}$.

\section{System programming}

In the system programming, this design consists of PLC programming and configuration simulation design. The combination of these two programs can well reflect the control process of the system. 


\subsection{PLC programming}

This design uses STEP7 programming software for PLC programming. The programming method used is the logic algebra design. It is to edit the PLC ladder diagram program according to the simplified logical expression ${ }^{5}$.

In this design, the PLC program needs to realize the following control actions. The first water pump starts and stops. When the frequency of the second frequency converter reaches the upper limit, the water pump is switched between frequency conversion and industrial frequency. Thirdly, when the pressure fluctuation of the pipeline is small, the water pump shall be alternated periodically. The fourth is to detect the liquid level, and the system can be stopped when the water supply level is too low.

PLC programming adopts modular programming. The main program OB1 is composed of seven functions or function blocks. Make the main program simple and visual for easy troubleshooting. The program in function FC1 is used to process the input analog quantity. The program in function FC2 is used for boot initialization. The program in function FC3 is the start-up program of the water pump motor. The program in function FC4 is the switch between frequency conversion and industrial frequency of the water pump motor. The program in function FC5 is an alarm program. The program in function FC6 is an analog output program. The program in function FC7 is a water pump alternate working program.

The main program OB1 part of the program design is shown in Fig.4.

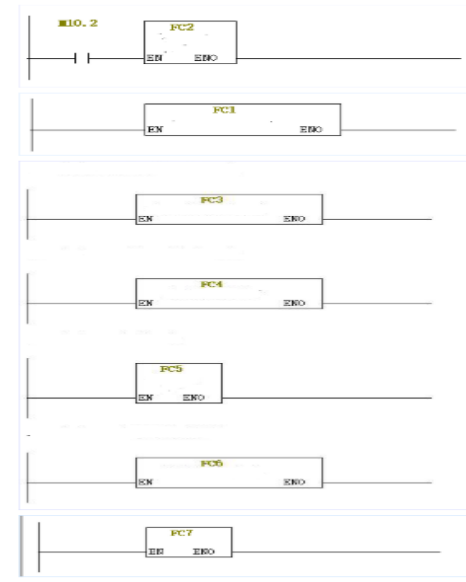

Fig.4. Main program OB1

\subsection{Configuration simulation design}

In this design, the design of the configuration screen of the upper computer system uses the KingView software produced by Beijing Yakong Company. The software is widely used in the current industrial field, and it has various functions such as process control design, field control, and resource management ${ }^{6}$.

The configuration screen designed by this system covers the main screen, real-time curve, alarm screen, historical data report, historical curve, and real-time data report. In the main configuration screen, the operator can start, stop and emergency stop the system. And can adjust the pressure of the pipe network. At the same time, it can monitor the running status of the water pump, the liquid level of the pool and the pressure of the outlet pipe network. On the main page, you can also perform a pump fault test. The main configuration screen is shown in Fig.5.

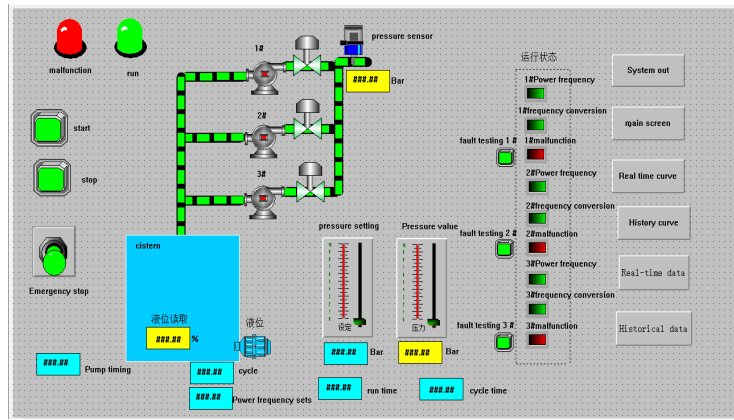

Fig.5. Main screen

\section{System Simulation}

After the system design is completed, the system can be simulated on the Kingview software.

In the simulation of this design, the main screen of the system can realize real-time monitoring of the operation of the automatic water supply system. In the real-time curve interface, the operator can intuitively monitor the pipe network pressure and pool water level in the system through real-time changes in the curve. It can be seen in the real-time curve simulation image that by changing the system water supply pressure setting value, the system will automatically adjust the actual water supply pressure to match the standard value. The real-time curve image is shown in Fig.6. 


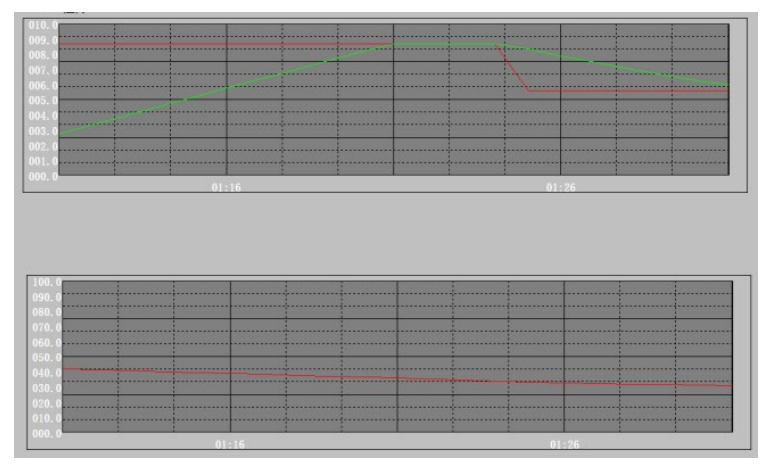

Fig.6. Real-time curve

In the historical curve interface, the operator can intuitively monitor the changes of the pipe network pressure and the water level of the pool in the historical time through the historical changes of the curve. After many simulations, the historical curve interface is shown in Fig. 7.

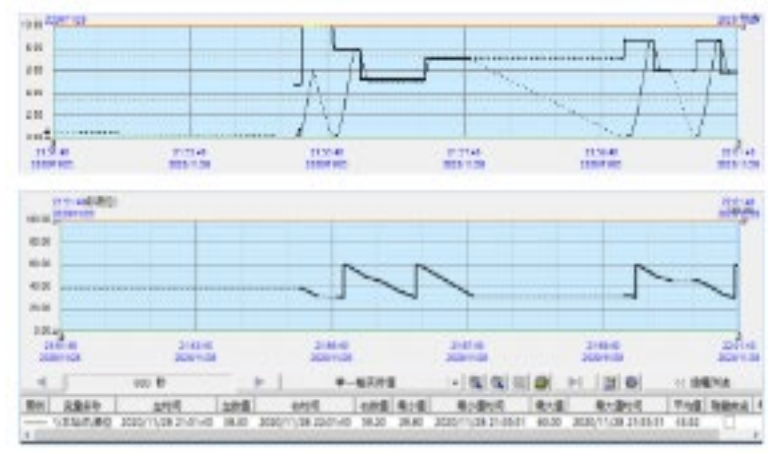

Fig.7. Historical curve

\section{Conclusion}

This design uses the KingView software combined with PLC and frequency converter to form an automatic water supply control system, replacing the previous valve control and manual water supply system. It is ensured that the pressure of the water supply pipe network will not drop during the time when the residents have more water. It also ensures that when the residential water is low, there will be no impact on the components of the entire system due to excessive pressure on the pipe network. It greatly slows down the aging of the water supply system components and extends the service life of the system.

The upper computer system in this design realizes the monitoring of the running status of the lower computer and the water pump. Can read the real-time data and historical data of the automatic water supply system. Monitor the changes in the operation of the water pump and the occurrence of system failures when the pressure of the water supply system fluctuates. The combination of KingView and PLC in this design makes the system program simple, integrated and highly transplantable.

\section{References}

1. Xiaoling Mo, Based on PLC the Automatic Constant Pressure Control Water Supply System Design, Applied Mechanics and Materials, 2014, 12: pp.1587-1590.

2. Tiewei Bu, The Overall Design of Frequency Conversion Water Supply Control System Based on PLC, Advanced Materials Research, 2014, 4: pp.1465-1468.

3. Yilin Qin, Siemens S7-300 PLC application technology, Electronic Industry Press, Beijing, China, 2007.

4. Yong Pan, Jian Wang, Quick commissioning of MICROMASTER440 inverter, Automation application, 2011, 1: pp.21-23.

5. Xuiling Lu, Several commonly used programmable controller ladder diagram design methods, Machine Tool Electric Apparatus, 2004, 10: pp.43-45.

6. Zhenfang Liu, Based on KingView configuration software and PLC water tower water level control system, Digital Communication World, 2019, 9: pp.85-86.

\footnotetext{
(C) The 2021 International Conference on Artificial Life and Robotics (ICAROB2021), January 21 to 24, 2021
} 